\title{
Österreichischer Dermatologen Preis 2015
}

In diesem Jahr wurden der Tumorforscher Dr. Igor Vujic von der University of California, San Francisco und dem Krankenhaus Rudolfstiftung in Wien und Dr. Doris Weiss von der Medizinischen Universität Wien ausgezeichnet. Der heuer bereits zum 43. Mal verliehene Österreichische Dermatologen Preis wurde am 4. Dezember in Wien, im Rahmen des President's Dinner anlässlich der 125jährigen Jubiläumsjahrestagung der Österreichischen Gesellschaft für Dermatologie und Venerologie (ÖGDV) überreicht.

Schon zum 43. Mal würdigt der Österreichischen Dermatologen Preis die bedeutendste Forschungsarbeit. Ausgezeichnet wurde heuer Dr. Igor Vujic für eine in Österreich, Italien und den USA durchgeführte Arbeit. Er konnte nachweisen, dass sich mit einer Wirkstoff-Kombination zweier Agentien bestimmte Tumorzellen hemmen lassen. Damit tun sich neue experimentelle Wege in der Grundlagen-Forschung verschiedener Krebsformen auf.

\section{Hemmen der NRAS-Stoffwechsel- wege}

Das mutierte NRAS-Protein - Neuroblastoma Ratten-Sarkom Onkogen spielt eine wichtige Rolle bei der Ausprägung verschiedener Krebsformen. Die direkte Hemmung des Onkogen-Proteins ist bisher gescheitert. Dr. Vujic applizierte nun eine Agentien-Kombination aus Metformin and Trametinib in Melanom-, Lungenkrebs- und Neuroblastom-Zellen, um die weiteren Stoffwechselwege des Tumorproteins in der Zelle zu blockieren. Beide Substanzen waren einzeln oder in Kombination mit anderen Agentien bekannt für ihre tumorhemmende Wirkung. Mit der Dual-Kombination konnte nun gezeigt werden, dass die downstream-Kaskaden des NRAS in den genannten Tumorzelllinien explizit gehemmt werden. In einem Transplantationsexperiment mit humanen Tumorzellen konnte auch in vivo ein Effekt festgestellt werden.

\section{Diagnose von Pemphigus vulgaris und P. folicaeus}

Dr. Doris Weiss von der Medizinischen UniversitätWien konnte mitimmun-pro- teinchemischen Methoden Pemphigus diagnostizieren. Dabei stellte sich heraus, dass in der Pemphigus-Diagnose das Immunoassay-Nachweisverfahren (ELISA) indirekter Immunfluoreszenz (IIE) deutlich überlegen ist.

Die zu den Autoimmunkrankheiten gehörenden Pemphigus-Varianten kommen insbesondere bei älteren Patienten vor. Die eher seltene blasenbildende Krankheit ist derzeit Gegenstand aktueller Forschung. Dr. Doris Weiss untersuchte die Diagnostik von zwei Pemphigus-Varianten, Pemphigus vulgaris und $P$. folicaeus, um die Diagnostik der beiden Hautkrankheiten zu verbessern. Die Wiener Wissenschaftlerin testete mittels zweier immunchemischer Methoden, dem Immunoassay ELISA und der indirekten Immunfluorenszenz (IIE) inwieweit beide Methoden für die Diagnostik geeignet sind. Ergebnis: Mit ELISA konnte die Krankheitsaktivität der beiden Pemphigus-Formen am besten abgebildet werden. Mit der indirekten Immunfluoreszenz IIE konnte die klinische Aktivität weit weniger akkurat diagnostiziert werden.

\section{Ältester Preis der ÖGDV}

Der Österreichische Dermatologen Preis für wissenschaftliche Arbeiten wird seit 1973 von Unilever Austria gestiftet. „Der Preis ist für Arbeiten mit Grundlagen-orientierten experimentellen Fragestellungen bestimmt, bei denen erkenntnistheoretische Aspekte im Vordergrund stehen, unabhängig von ihrer unmittelbaren Anwendbarkeit. Die Auszeichnung ist der älteste von der Österreichischen Gesellschaft für Dermatologie und Venerolo-

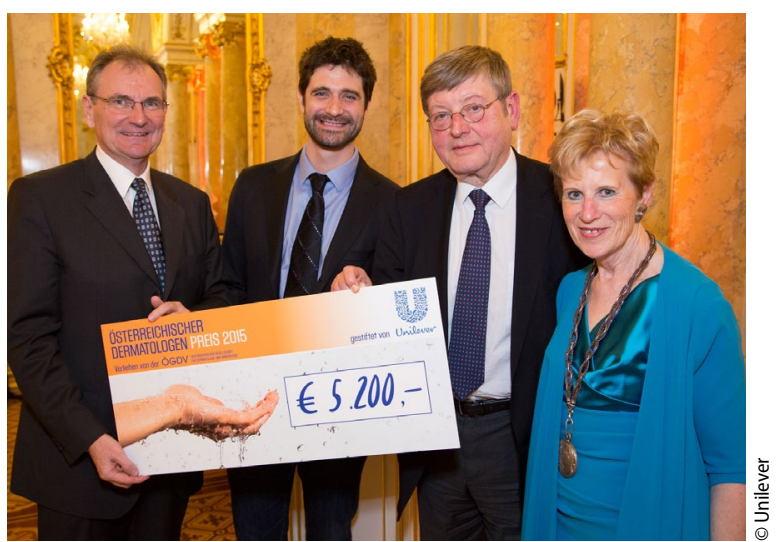

$\Delta$ (v.li.n.re.): Ing. Gerhard Gribl (Unilever Austria), Dr. Igor Vujic (Preisträger Dermatologen Preis 2015), Prof. Dr. Georg Stingl (Vorstandsmitglied ÖGDV), Prof. Dr. Angelika Stary (Präsidentin des ÖGDV). Prof. Dr. Georg Stingl nahm den Preis in Vertretung für Dr. Doris Weiss entgegen

gie verliehene und von Unilever Austria gestiftete Preis", so die Präsidentin der ÖGDV, Prof. Dr. Angelika Stary.

Der mit 5200 Euro dotierte Österreichische Dermatologen Preis wurde am 4. Dezember im Wiener Palais Coburg, im Rahmen des President's Dinner anlässlich der 125jährigen Jubiläumsjahrestagung der ÖGDV überreicht. „Wir freuen uns sehr über die eingereichten, hervorragenden Arbeiten. Beide Preisträger sind würdige Nachfolger in der Tradition von Dr. Moriz Kaposi, der 1890 - also genau vor 125 Jahren - die Österreichische Gesellschaft für Dermatologie und Venerologie gründete", so Prof. Dr. Angelika Stary abschließend.

hautnah $2016 \cdot 15: 9$

DOI 10.1007/s12326-016-0180-x

Online publiziert: 28. Januar 2016

๑) Springer-Verlag Wien 2016
Quelle: Presseaussendung BuzzDriver 